\title{
Mediation of Mothers' Anxiety and Parenting in Children's Behavior Problems during COVID-19
}

\author{
Keren Hanetz-Gamliel ${ }^{1} \cdot$ Sigal Levy ${ }^{2}$ • Daphna G. Dollberg ${ }^{1}$ \\ Accepted: 9 September 2021 / Published online: 23 September 2021 \\ (c) The Author(s), under exclusive licence to Springer Science+Business Media, LLC, part of Springer Nature 2021
}

\begin{abstract}
The outbreak of COVID-19 is affecting the lives of millions of families around the world. The current study was carried out in Israel, following the pandemic's initial outbreak and during the resulting enforced quarantine, confining parents and children to their homes. A sample of 141 Israeli mothers with at least one child between the ages of 3 and $12(M=6.92$, $\mathrm{SD}=2.55)$ participated as volunteers. About half the sample $(50.7 \%)$ consisted of girls. Most mothers were cohabiting with a spouse (93\%). Mothers completed online questionnaires about their perceptions about the health and economic threats of COVID-19, availability of social support, their anxiety symptoms, hostile/coercive and supportive/engaged parenting behavior, and their children's behavior problems. Results showed expected significant associations between the mothers' reports about having little social support, their anxiety symptoms, hostile/coercive and supportive/engaged parenting behavior, and children's externalizing problems. Likewise, expected significant associations were found between mothers' perceptions about the health and economic threats of COVID-19, their anxiety symptoms, hostile/coercive parenting behavior, and children's internalizing and externalizing problems. Importantly, maternal anxiety and hostile/coercive parenting behavior mediated the associations between lack of support, negative perceptions about the health and economic threats of COVID-19, and children's behavior problems. These findings stress the importance of mothers' mental health and parenting behaviors for children's socioemotional adaptation in the context of COVID-19. Implications of the findings for family interventions intended to help parents and children at this time are suggested.
\end{abstract}

Keywords COVID-19 $\cdot$ Mother's anxiety $\cdot$ Maternal behavior $\cdot$ Child behavior problems $\cdot$ Social distancing

\section{Highlights}

- Maternal anxiety mediates the associations between COVID-19's contextual features and children's behavior problems.

- Hostile/coercive parenting behavior mediates the associations between COVID-19's contextual features and children's behavior problems.

- Mothers' mental health and parenting are crucial for children's socioemotional adaptation in the context of COVID-19.

- Social distancing policies may lead to lack of social support, which is a risk for mothers' anxiety and parenting.

Since December 2019, the lives of millions of families around the world have changed dramatically and instantaneously due to the outbreak of the COVID-19 pandemic.

Keren Hanetz-Gamliel

kerenhan@mta.ac.il

1 School of Psychology, Academic College of Tel Aviv Yaffo, Jaffa, Israel

2 Statistics Education Unit, Academic College of Tel Aviv-Yaffo, Jaffa, Israel
In many countries, a partial or full quarantine was enforced in an attempt to halt the virus's spread. In Israel, the quarantine included a partial lockdown, restrictions on outdoor movement, strict limitations on social gatherings, the complete shutdown of the educational system, restrictions on leisure activities and a significant growth in remote working from home. These dramatic changes affected almost every aspect of families' lives and likely shaped their everyday routines and emotional climate.

Previous research showed that children are among the most vulnerable groups affected by disasters (Silverman \& La Greca, 2002). Indeed, initial reports from around the 
world have noted the adverse emotional reactions of children to COVID-19. For example, in China, researchers reported fears, inattention, irritability, regression and clinginess among children (Jiao et al., 2020). Likewise, Italian and Spanish parents noted difficulties in concentration, irritability, restlessness, nervousness and worries in their children (Orgilés et al., 2020; Romero et al., 2020). Still, the reports did not address the questions of how and in what ways COVID-19 relates to children and parents.

To fill this gap in the literature, we explored how the contextual stressors of COVID-19 are linked directly and indirectly to children's adaptation via their mothers' mental health and parenting behavior. To do so, we used the family stress model (Conger \& Conger, 2002; Conger et al., 2010), which argues that stressors influence children's adjustment through mediating pathways such as parents' psychological distress and parenting (Masarik \& Conger, 2017), as our theoretical framework.

\section{Mothers' Perceptions about the Threats Related to COVID-19}

Researchers have long noted that subjective assessments about the risks involved in a disaster affect people's emotional and behavioral reactions even more than the exposure to the disaster itself (Slovic, 1987). Thus, threat perceptions are central in explaining reactions and adaptation to disasters. Parents' perceptions of and reactions to a specific traumatic event are crucial for parenting (Cobham et al., 2016) and determine their children's wellbeing (Juth et al., 2015) over and above the events themselves.

Accordingly, studies have documented that parents' subjective evaluations of the implications of COVID-19 are associated with their children's adjustment (Saddik et al., 2020), the parents' anxiety (Malhotra et al., 2020), and the quality of their parenting (Chung et al., 2020; Romero et al., 2020). We explored a possible direct association between mothers' perceptions about the health and financial threats of COVID-19 and their children's behavior problems as well as the indirect links via the mothers' anxiety and maternal behavior.

\section{Lack of Social Support for Mothers during COVID-19}

The outbreak of COVID-19 was followed by a worldwide policy of social distancing, which included significant limitations on social and family face-to-face interactions, resulting is social isolation and limited social support for parents and children (e.g., Imran et al., 2020; Romero et al., 2020). Play dates, grandparents' visits and out-of-home childcare arrangements were banned, resulting in many families losing their usual social network and childcare support (Griffith, 2020). In Israel, working parents tend to depend a great deal on childcare assistance. A national survey showed that among families of children aged 6-13, $18.8 \%$ of parents depended on extended family childcare and $25.1 \%$ relied on paid, non-kin childcare arrangements (Israel Central Bureau of Statistics, 2008). Moreover, generational family solidarity is a key component of Israeli life (Katz et al., 2015). Grandparents often reside nearby and provide childcare support, and serve as a source of social and emotional support for parents and grandchildren. Thus, the COVID-19 social distancing policy left many Israeli families isolated and without their usual childcare and kin support, affecting both parents and children.

Researchers have documented that lack of available social support is associated with increased risk for mental health problems in mothers (Parkes et al., 2015). A large longitudinal Scottish study reported a positive association between lack of support for mothers and children's internalizing and externalizing behavior, which was mediated by maternal distress and dysfunctional parenting (Parkes \& Sweeting, 2018). Similarly, during COVID-19, parents' perceptions about unavailability of support were associated with increased stress and higher risks of child abuse (Brown et al., 2020). Based on this evidence, we argue that the dramatic reduction in daily social and familial encounters during COVID-19 likely resulted in mothers feeling isolated and lacking their usual social support. We explore the link between this loss of usual support and mothers' reported anxiety and parenting behavior during the COVID-19 outbreak.

\section{Mothers' Anxiety, Parenting and Children's Behavior Problems during COVID-19}

Studies from around the world have reported an increase in anxiety among adults during the COVID-19 outbreak (Luo et al., 2020; Mazza et al., 2020) with women reporting the highest levels of anxiety (Saddik et al., 2020). Maternal anxiety is considered a risk factor for children's development, predicting the latter's emotional, cognitive and behavioral difficulties (Behrendt et al., 2020; Gibler et al., 2018). Studies we conducted in Israel prior to COVID-19 showed that parents' anxiety symptoms were positively linked with reports of their children's externalizing and internalizing behaviors (MASKED, 2018; MASKED, 2019). Moreover, according to the family stress model (Masarik \& Conger, 2017), in the presence of external threats, primary caregivers' emotional reactions, particularly their anxiety levels, play a significant role in children's responses. Thus, not surprisingly, recent research found that parents' anxiety symptoms during COVID-19 were 
associated with increases in children's problems, specifically their internalizing problems (Saddik et al., 2020; Whittle et al., 2020).

Maternal anxiety is a risk factor for negative parenting (Hentges et al., 2019) and reduced maternal warmth (Epkins \& Harper, 2016), leading to preschoolers' behavior problems, especially their externalizing behavior (Song, 2018). Given the elevation in maternal anxiety and children's behavior problems during COVID-19, we propose looking at the role of maternal anxiety and related parenting behavior in mediating these links.

\section{Hostile and Supportive Parenting and Children's Behavior Problems during COVID-19}

Hostile parenting is defined as the parent's negative affect, anger, harshness, criticism, disapproval (Connor \& Rueter, 2006) and indifference or the use of coercive behavior towards the child (Lovejoy et al., 1999). In contrast, supportive parenting is defined as parental warmth, affection, interest in (Connor \& Rueter, 2006) and acceptance of the child, and engagement in shared activities (Lovejoy et al., 1999). Mounting empirical evidence indicates a positive link between hostile, harsh maternal parenting and children's behavior problems (Schwartz et al., 2014; Yap et al., 2014) as well as adolescents' poor adjustment to school (Weymouth et al., 2016). In contrast, positive parenting strategies, specifically supportive parenting and maternal warmth, are linked to fewer externalizing and internalizing behaviors (Rothenberg et al., 2020).

Negative parenting behavior is common in times of stress and crisis, and is linked with children's reactions and behavior (Cobham et al., 2016). Specifically, negative parenting behavior, such as corporal punishment and hostile, anxious and overprotective parenting styles, predict children's emotional and behavior problems after a natural disaster (Kelley et al., 2010) and exposure to war (Halevi et al., 2017). Interestingly, whereas the literature regarding the risks associated with negative parenting behavior is well documented, the advantages of positive parenting for children following crises are inconclusive. While some reported a beneficial association between parents' positive behavior such as acceptance, support, communication and involvement and children's adjustment (Gewirtz et al., 2008), other studies failed to find such links (Gil-Rivas \& Kilmer, 2013).

When it comes to COVID-19, accumulating evidence indicates significant associations between negative parenting behaviors and a decline in children's mental health, including an increase in the rates of internalizing and externalizing problems (Romero et al., 2020; Whittle et al., 2020). On the other hand, supportive and engaged parenting during this period was associated with reductions in the children's level of worry, their greater tolerance of uncertainty (Shen et al., 2020) and their increased wellbeing (Neubauer et al., 2020). Furthermore, anecdotal, clinical and empirical evidence point to variations in children's and families' adjustment to the pandemic, where some thrived and others crumbled (Clements et al., 2020; Günther-Bel et al., 2020). These variations can be explained by the family stress model (Masarik \& Conger, 2017), which argues that the links between COVID-19 and children's adaptation may be mediated by factors within the family. Indeed, some of the latest works addressing children and family during COVID-19 proposed mediation models linking the pandemic with parents' emotional state, parenting and children's outcomes. For example, a Spanish study reported a mediation model in which parents' anxiety symptoms were associated with increased parental distress. This distress, in turn, was linked directly to children's emotional problems, hyperactivity and conduct problems, and indirectly via a lack of focused and structured parenting (Romero et al., 2020).

\section{The Current Study}

Our aim was to explore and broaden our understanding of the role of parenting in the unique context of the COVID-19 crisis. Inspired by the evolving understanding that parenting is a vital factor to consider in relation to children's socioemotional adaptation especially in times of emergency and guided by the family stress model as its theoretical framework (Masarik \& Conger, 2017), we proposed and tested a model linking mothers' perceptions about the threat and the unavailability of support from family and friends with their anxiety and parenting behavior. In turn, we posited that these factors would be associated with children's internalizing and externalizing behavior.

To test this model, we gathered data from Israeli mothers from mid-March until the end of April 2020. At that time the virus was rapidly spreading across the country, resulting in an enforced quarantine. Based on the emerging data on variations in families' adaptation to the pandemic (Clements et al., 2020; Günther-Bel et al., 2020), we hypothesized that in some families, mothers' perceptions about the medical and economic hazards associated with the pandemic and their limited face-to-face social and emotional support might be linked positively and directly with the children's behavior problems. Alternatively, for some mothers, heightened perceptions about the threat and lack of support might indirectly be linked to more anxiety, which, in turn, would be associated with more impatient and hostile behavior in dealing with their children. It is also possible that some mothers may have benefitted from the quarantine, 
enjoying the shared family time, and the increased paternal involvement due to fathers spending more time at home. For these mothers, these same contextual features of COVID-19 may be linked to less anxiety and more benevolent maternal parenting behavior, which in turn, might be associated with fewer behavior problems in their children.

We posited two hypotheses:

H1. Mothers' perceptions about COVID-19 as a threat and the resulting lack of social support will be (a) positively associated with their anxiety symptoms and their children's internalizing and externalizing problems, and (b) positively associated with hostile/coercive maternal parenting behavior and negatively associated with supportive/engaged parenting behavior.

H2. The positive links between mothers' perceptions about COVID-19 as a threat and the lack of social support and children's behavior problems will be mediated by the mothers' anxiety symptoms and parenting. Therefore, (a) mothers who regard COVID-19 as more alarming and those who report having less social support will report more anxiety. Greater maternal anxiety will be linked with children's internalizing and externalizing problems either directly or indirectly via hostile/coercive parenting behavior; (b) mothers who regard COVID-19 as less alarming and those who report having more social support will report less anxiety. Less maternal anxiety will be negatively linked with children's internalizing and externalizing problems either directly or via supportive/engaged parenting behavior.

\section{Method}

\section{Participants}

Two hundred and sixty-two Israeli mothers of children between the ages of 3 and 12 years old volunteered for the study. Of the 262, 141 mothers completed all of the study's questionnaires and comprised the final sample. Independent sample $t$-tests and $\chi^{2}$ tests found no significant differences between those who completed the questionnaires entirely and those who did not in their main demographic variables such as age, education, income and household composition. The children ranged in age from 3 to 12.67 years old $\left(M_{\text {age }}=6.92, \mathrm{SD}_{\text {age }}=2.55\right)$. Girls comprised $50.7 \%$ of the sample. The majority of the children (58.3\%) were firstborn, and all attended public schools. The mothers' mean age was 39.13 years old $\left(\mathrm{SD}_{\mathrm{age}}=5.78\right)$, with $57.2 \%$ of them having a post-secondary education. A majority of the mothers (93\%) were cohabiting with a spouse. Many mothers $(90.8 \%)$ indicated that they were in good or very good physical health before COVID-19 and none reported being sick with COVID-19 prior to or during the time of the study. The great majority of mothers indicated above average $(57.1 \%)$ and average (40\%) pre-COVID-19 family income according to Israeli standards.

\section{Procedure}

Recruitment for the current study was carried out in the midst of the outbreak of the COVID-19 pandemic and during the enforcement of quarantine in Israel (from midMarch until the end of April 2020). During these months, advertisements were posted on parents' social networks and the link to the questionnaire was spread through the snowball technique. Mothers were asked to consider only one of their children in the specified age range when completing the questionnaires. Data collection ended when the quarantine ended, and the school system reopened under certain restrictions. The XXX's (MASKED) Ethics Committee approved the study (ref. 2020079). All participants signed an informed consent prior to participation. Participation was anonymous and voluntary. There was no reward or compensation for participation.

\section{Measures}

\section{Perceptions about the threat of COVID-19}

We measured mothers' subjective perceptions about the threat of the coronavirus pandemic using a 3-item questionnaire designed specifically for the current study. The questions addressed the mothers' assessment of the long-term implications of the pandemic for their physical and mental health, and the family's economic situation on a 5-point Likert-type scale, ranging from 1 (destructive implication) to 5 (no implication). The mean score was reverse coded to correspond to the other variables in the study, so that a higher score indicated greater perceived threats. Cronbach's alpha was 0.65 .

\section{Lack of social support during COVID-19}

To assess their sense of being supported, the mothers answered two questions: "To what extent do you feel you received support from extended family members during the quarantine?" and "To what extent do you feel you received support from friends or neighbors during the quarantine?" that were rated on a 3-point scale ranging from 1 (very little support) to 3 (a lot of support). The mean score was reverse coded to reflect a lack of social support and to correspond to the other variables in the study, so that a high score reflected a lack of social support. Cronbach's alpha was 0.65 .

\section{Mothers' anxiety symptomatology}

We used the 6-item anxiety subscale of the Brief Symptom Index (BSI; Derogatis, 1993) to assess the mothers' reported 
anxiety symptoms. The BSI is a widely used, psychometrically validated, reliable self-report questionnaire (Derogatis \& Melisaratos, 1983) comprised of 53 items that measure distress. Respondents were asked to answer questions such as: "Over the last month, to what extent did you feel fearful?" on a 5-point Likert-type scale, ranging from 0 (not at all) to 4 (extremely). Higher scores reflect greater psychopathology. Internal consistency of the anxiety subscale in the current study was good (Cronbach's alpha $=0.79$ ).

\section{Maternal behavior}

The Parent Behavior Inventory (PBI, Lovejoy et al., 1999) was used to evaluate the mothers' parenting behavior. The PBI is a 20-item measure of parenting behavior, rated on a 6-point Likert scale from 0 (not at all or never) to 5 (always) that produces two independent scales: supportive/engaged and hostile/coercive. The supportive/engaged parenting dimension consists of 10 items (e.g., "I have pleasant conversations with my child"). The hostile/coercive dimension similarly consists of 10 items (e.g., "I say mean things to my child that makes him/her feel bad"). The scale was designed for and has been used with parents of preschool-age and school-age children (Condone et al., 2019; Lovejoy et al., 1999). In the current study the two scales had adequate internal consistency (Cronbach's alpha $=0.78$ and 0.81 for the hostile/coercive and the supportive/engaged scales, respectively).

\section{Children's behavior problems}

We measured this factor using the parental Child Behavior Checklist questionnaire. This questionnaire has two versions, depending on the child's age. For children from 3 to 5 , mothers completed the CBCL 11/5- 5-year-old (Achenbach \& Rescola, 2000), and for children 6 and older, they completed the CBCL 6-18 years old (Achenbach \& Rescola, 2001). In both versions, items are presented on a 3-point Likert-type scale, ranging from 0 (not true), through 1 (somewhat or sometimes true), to 2 (very or often true). The CBCL for preschoolers contains 99 items, such as "can't sit still, is restless, or hyperactive." The CBCL for school-age children contains 113 items, such as "not liked by other kids." The two versions yield two clusters: internalizing behavior and externalizing behavior. In the current study, both clusters demonstrated good internal consistency (for younger children: internalizing behavior: Cronbach's alpha $=0.88$; externalizing behavior: Cronbach's alpha $=$ 0.90; for older children: internalizing behavior: Cronbach's alpha $=0.82$, externalizing behavior: Cronbach's alpha $=$ 0.91). The CBCL has standardized scores with Israeli norms for each age category (Zilber et al., 1994). There were no significant correlations between the children's age and the raw scores of their internalizing or externalizing behavior in any age group. Thus, we converted the raw scores to $t$-scores for each age group and used them across the two age groups.

\section{Results}

Prior to testing the study's hypotheses, we conducted preliminary analyses considering associations between various demographics and the study's variables. Pearson's correlation coefficients were used to test for associations between the study's variables and the demographic characteristics of the participants. The results revealed that the child's gender was associated with the mothers' reports of their children's internalization problems $(r=0.18, p=0.03)$. Specifically, mothers reported more internalizing behaviors in their daughters $(M=52.51, \mathrm{SD}=10.70)$ than their sons $(M=$ $48.80, \mathrm{SD}=9.15$ ). In addition, the child's age was significantly and negatively associated with the mothers' reports of supportive/engaged parenting $(r=-0.16, p=$ 0.04 ), so that mothers of older children reported fewer supportive and engaged parenting behaviors. Several studies have indicated that the children's age and gender contribute to their reactions to COVID-19 (e.g., Imran et al., 2020; Romero et al., 2020; Zhou et al., 2020). Thus, we included the child's gender and age as covariates in further analyses.

To test H1, we computed Pearson's $r$ correlations to investigate associations among the study's variables. Table 1 presents the descriptive information (means and standard deviations or counts and percentages, as appropriate) and correlations among the study's variables controlling for the child's gender and age. In support of H1a, the mothers' perceptions about the threat of COVID-19 and their sense of a lack of social support were significantly and positively associated with their reports of their anxiety symptoms. Mothers' perceptions about the threat of COVID-19 and their reports of lack of social support were also significantly and positively associated with their children's externalizing problems. Furthermore, a significant positive relationship between the mothers' perceptions about the threat of COVID-19 and their children's internalizing problems was found. However, no similar association was found between the mothers' reports of lack of social support and their children's internalizing problems.

With regard to positive and negative maternal parenting (H1b), as hypothesized, we found a significant positive relationship between the mothers' reports of lack of social support and hostile/coercive parenting behavior, and a significant negative association with supportive/engaged parenting behavior. The mothers' perceptions about the threat of COVID-19 had a significant relationship with hostile/ coercive parenting behavior, but not with supportive/ engaged parenting behavior. Furthermore, the mothers' 


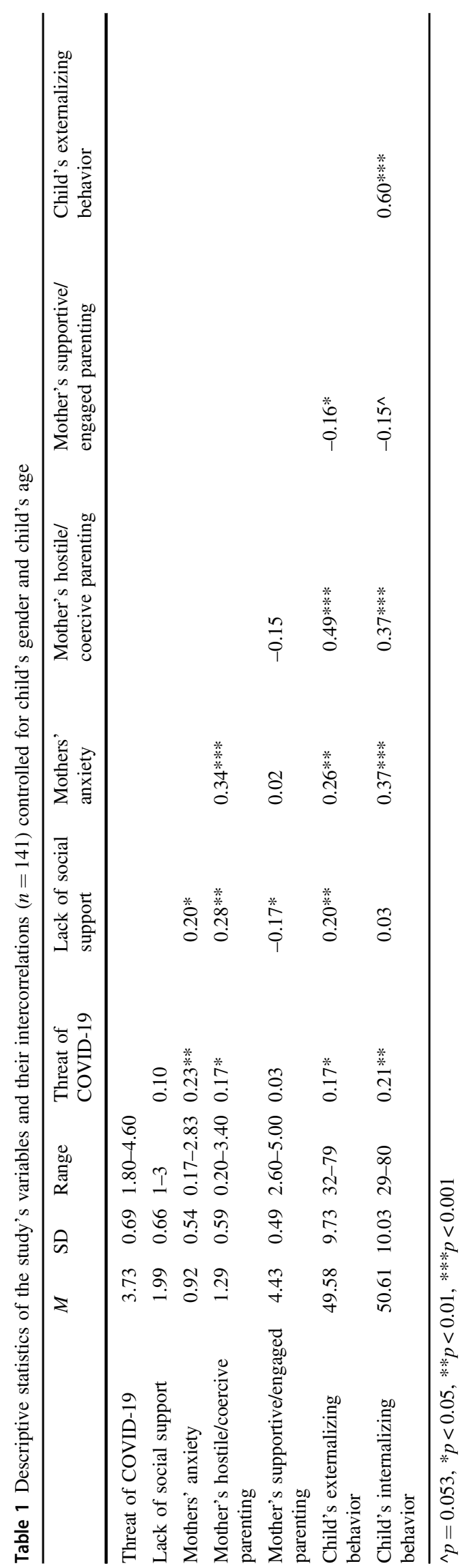

reports of their anxiety symptoms were positively associated with their children's externalizing and internalizing problems and with the mothers' hostile/coercive parenting behavior, but not with their supportive/engaged parenting. Finally, hostile/coercive maternal parenting behavior was positively associated with children's externalizing and internalizing problems, whereas supportive/engaged parenting was correlated negatively with the children's externalizing problems and nearly significantly with their internalizing problems. In sum, H1 was largely supported.

To test H2, we used path analysis with 1000 biascorrected bootstrapping. Two models were tested separately, one consisting of negative maternal behavior such as hostile/coercive parenting, and one consisting of positive maternal behavior such as supportive/engaged parenting. Goodness-of-fit was assessed using the $\chi^{2}$, root-meansquare error of approximation (RMSEA), and the comparative fit index (CFI). According to suggestions by $\mathrm{Hu} \&$ Bentler (1999), RMSEA values less than or equal to 0.06, and CFI values of 0.95 or higher are considered indicators of a good model fit, whereas a RMSEA smaller than 0.08, and a CFI larger than 0.90 indicate an adequate model fit. We analyzed the data using IBM SPSS v25 and AMOS v25 (Arbuckle, 2019). Given our earlier finding about the significant correlations between the child's age and gender and the study's variables and previous evidence regarding their links with children's adaptation during COVID-19, we included these two factors as covariates in the models. Table 2 indicates the indirect path coefficients for the associations between the mothers' perceptions about the threat of COVID-19, their reports about lack of social support and their children's externalizing and internalizing problems in the two models via mothers' anxiety and maternal behavior (either hostile/coercive or supportive/ engaged parenting behaviors).

The first path analysis model with mothers' anxiety and hostile/coercive maternal parenting as mediators showed an adequate model fit $\left(\chi^{2}(4)=6.58, p=0.16, \mathrm{CFI}=0.99\right.$, RMSEA $=0.06)$ and accounted for $27 \%$ and $28 \%$ of the externalizing and internalizing problems, respectively. Figure 1 depicts the links between the model's variables. While not presented in the figure for the sake of visual clarity, we did establish that the child's age had a significant effect on the child's internalizing behavior $\beta=0.23,95 \% \mathrm{CI}=$ $[0.09,0.36], p=0.001$, as did the child's gender $\beta=-0.15$, $95 \% \mathrm{CI}=[-0.28,-0.02], p=0.03$. There were no direct effects of the mothers' perceptions about the threat of COVID-19 or their reports about lack of social support on their children's externalizing and internalizing problems. Mothers' anxiety and hostile/coercive parenting behaviors significantly mediated the links between the mothers' perceptions about the threat of COVID-19 and the children's externalizing and internalizing behavior problems, as did 
Table 2 Standardized indirect effects of mothers' perceptions of the threat of COVID-19 and reports about lack of social support and children's behavior via mothers' anxiety and mothers' positive and negative parenting

\begin{tabular}{|c|c|c|c|c|c|c|}
\hline & \multicolumn{3}{|c|}{$\begin{array}{l}\text { Mother's hostile/coercive } \\
\text { parenting }\end{array}$} & \multicolumn{3}{|c|}{$\begin{array}{l}\text { Mother's supportive/ } \\
\text { engaged parenting }\end{array}$} \\
\hline & $\beta$ & $95 \% \mathrm{CI}$ & $p$ & $\beta$ & $95 \% \mathrm{CI}$ & $p$ \\
\hline Threats of COVID-19 $\rightarrow$ mother's anxiety $\rightarrow$ child's externalizing behavior & 0.02 & $-0.08,0.01$ & 0.23 & 0.05 & $-0.13,0.01$ & 0.03 \\
\hline Threats of COVID-19 $\rightarrow$ mother's anxiety $\rightarrow$ child's internalizing behavior & 0.06 & $-0.14,0.00$ & 0.04 & 0.08 & $-0.16,0.00$ & 0.05 \\
\hline Threats of COVID-19 $\rightarrow$ maternal behavior $\rightarrow$ child's externalizing behavior & 0.03 & $-0.10,0.03$ & 0.34 & 0.00 & $-0.05,0.02$ & 0.51 \\
\hline Threats of COVID-19 $\rightarrow$ maternal behavior $\rightarrow$ child's internalizing behavior & 0.02 & $-0.08,0.02$ & 0.28 & 0.00 & $-0.05,0.02$ & 0.55 \\
\hline $\begin{array}{l}\text { Threats of COVID-19 } \rightarrow \text { mother's anxiety } \rightarrow \text { maternal behavior } \rightarrow \text { child's externalizing } \\
\text { behavior }\end{array}$ & 0.03 & $-0.06,00$ & 0.03 & 0.00 & $0.00,0.02$ & 0.38 \\
\hline $\begin{array}{l}\text { Threats of COVID--19 } \rightarrow \text { mother's anxiety } \rightarrow \text { maternal behavior } \rightarrow \text { child's internalizing } \\
\text { behavior }\end{array}$ & 0.02 & $-0.05,0.01$ & 0.02 & 0.00 & $0.00,0.02$ & 0.36 \\
\hline Lack of social support $\rightarrow$ mother's anxiety $\rightarrow$ child's externalizing behavior & 0.02 & $-0.07,0.01$ & 0.19 & 0.04 & $-0.10,-0.01$ & 0.01 \\
\hline Lack of social support $\rightarrow$ mother's anxiety $\rightarrow$ child's internalizing behavior & 0.05 & $-0.12,-0.01$ & 0.01 & 0.07 & $-0.12,-0.02$ & 0.02 \\
\hline Lack of social support $\rightarrow$ maternal behavior $\rightarrow$ child's externalizing behavior & 0.09 & $-0.17,-0.03$ & 0.01 & 0.03 & $-0.08,0.00$ & 0.08 \\
\hline Lack of social support $\rightarrow$ maternal behavior $\rightarrow$ child's internalizing behavior & 0.06 & $-0.12,-0.02$ & 0.01 & 0.03 & $-0.10,0.00$ & 0.07 \\
\hline $\begin{array}{l}\text { Lack of social support } \rightarrow \text { mother's anxiety } \rightarrow \text { maternal behavior } \rightarrow \text { child's externalizing } \\
\text { behavior }\end{array}$ & 0.02 & $-0.06,0.00$ & 0.01 & 0.00 & $0.00,0.01$ & 0.34 \\
\hline $\begin{array}{l}\text { Lack of social support } \rightarrow \text { mother's anxiety } \rightarrow \text { maternal behavior } \rightarrow \text { child's internalizing } \\
\text { behavior }\end{array}$ & 0.02 & $-0.05,00$ & 0.00 & 0.00 & $0.00,0.01$ & 0.28 \\
\hline
\end{tabular}

Maternal behavior refers to either the mother's hostile/coercive or supportive/engaged parenting behavior

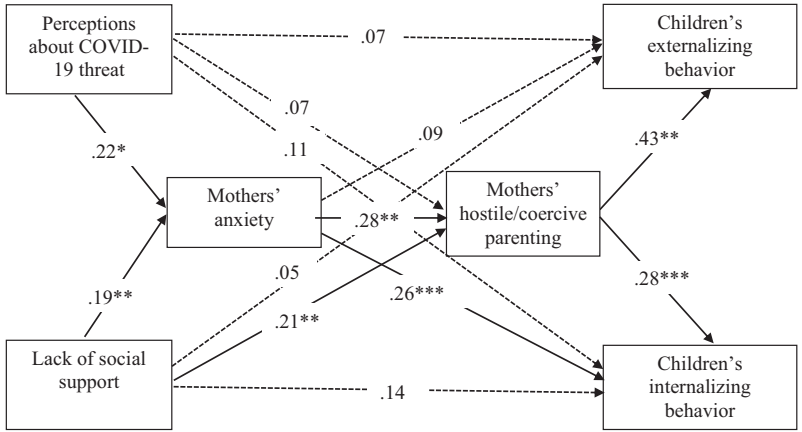

Fig. 1 Mediation model of the mothers' perceptions about the threat of COVID-19 and lack of social support and their children's behavior problems via the mothers' anxiety and hostile/coercive parenting. Numbers above the lines are standardized coefficients. While not presented in the figure, we included the child's age and gender in this model as covariates. In addition, we accounted for the intercorrelations between the contextual variables related to COVID-19, as well as for the intercorrelations between the error terms of the children's behavior variables. ${ }^{*} p<0.05 ; * * p<0.01$

the mothers' reports about lack of social support (see Fig. 1 and Table 2). In other words, mothers who regarded COVID-19 as more alarming, and those who felt they had less support at that time, reported more anxiety symptoms. Their anxiety, in turn, predicted more hostile/coercive behaviors which were linked with more internalizing and externalizing behavior problems in their children. Moreover, hostile/coercive maternal behavior in and of itself mediated the link between the mothers' reports of lack of support and their children's externalizing and internalizing problems. Additionally, the mothers' anxiety mediated the links between their reports about lack of support and their perceptions about the threat of COVID-19, but only in the case of internalizing problems in their children. Thus, mothers who regarded COVID-19 as more alarming and felt they had less social or family support tended to be more anxious. This anxiety was linked with more reports about children's internalizing problems, regardless of the mothers' parenting behavior.

The model predicting children's behavior problems from their mothers' perceptions about the threat of COVID-19 and their reports about lack of social support mediated by the mothers' anxiety and supportive/engaged parenting proved adequate in its fit $\left(\chi^{2}(3)=2.63, p=0.45, \mathrm{CFI}=\right.$ 1.00$, RMSEA $=0.00)$. The model accounted for $15 \%$ and $26 \%$ of the externalizing and internalizing behavior, respectively. The path model is presented in Fig. 2 and Table 2. As with Fig. 1, for the sake of visual clarity, we did not provide the children's age and gender in the model. Nevertheless, the child's age had a significant effect on supportive/engaged parenting $\beta=-0.24,95 \% \mathrm{CI}=[-0.39$, -0.11 ], $p=0.01$ and the child's internalizing behavior $\beta=$ $0.20,95 \% \mathrm{CI}=[0.05,0.35], p=0.02$. The child's gender also had a significant effect on the child's internalizing behavior $\beta=-0.19,95 \% \mathrm{CI}=[-0.31,-0.04], p=0.01$. Similar to Model 1, we found no direct links between the mothers' perceptions about the threat of COVID-19 and lack of support, and behavior problems in their children. 


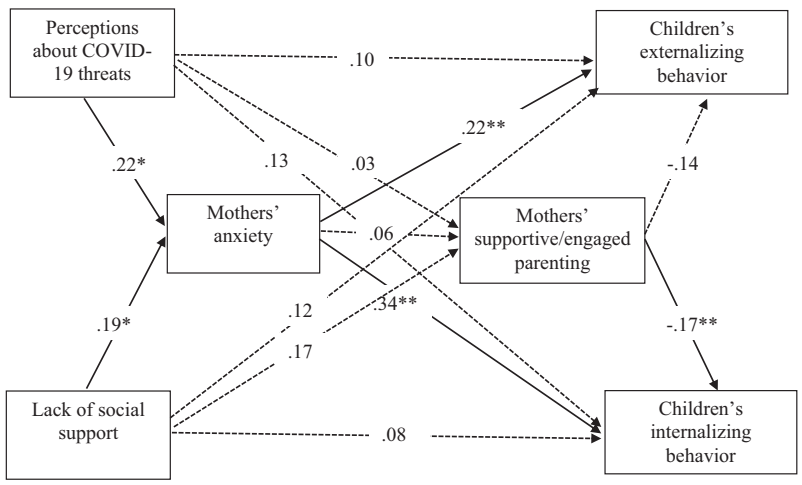

Fig. 2 Mediation model of the mothers' perceptions about the threat of COVID-19 and lack of social support and their children's behavior problems via the mothers' anxiety and supportive/engaged parenting. Numbers above the lines are standardized coefficients. While not presented in the figure, we included the child's age and gender in this model as covariates. In addition, we accounted for the intercorrelations between the contextual variables related to COVID-19, as well as for the intercorrelations between the error terms of the children's behavior variables. $* p<0.05 ; * * p<0.01$

However, significant indirect links were evident between the mothers' perceptions about the threat of COVID-19 and lack of social support, and their children's externalizing and internalizing problems via the mothers' anxiety. The indirect effects through supportive/engaged maternal parenting were close to significant when predicting the child's externalizing behavior $(\beta=0.03,95 \% \mathrm{CI}=[-0.08,0.00]$, $p=0.08)$ and internalizing behavior $(\beta=0.03,95 \% \mathrm{CI}=$ $[-0.10,0.00], p=0.07)$ from the mothers' reports about lack of social support. Paths that involved the two mediators consecutively (mothers' anxiety and supportive/engaged parenting) were not significant. Thus, in the case of supportive/engaged parenting, the mothers' anxiety had a major mediating effect on the link between the mothers' perceptions about the threat of COVID-19 and lack of social support, and their children's externalizing and internalizing behavior.

\section{Discussion}

Our findings join a greatly needed and rapidly emerging body of research documenting the links between COVID-19 and the family, particularly mothers' parenting behavior and children's socioemotional adaptation. Guided by the family stress model (Masarik \& Conger, 2017), our study sought to identify the COVID-19 related contextual features and parental risks as well as protective factors accounting for the adaptation of mothers and their children to the ongoing pandemic crisis.

As hypothesized, we found that mothers' subjective experiences about the threat posed by COVID-19 and their reports of lack of social and family support resulting from the enforced quarantine were positively associated with the mothers' anxiety symptoms and their children's behavior problems. The mothers' subjective sense of threat and lack of support were also positively associated with their negative parenting, reflected in more hostile and coercive behaviors. Furthermore, the lack of social and family support was significantly and negatively associated with the mothers' positive parenting behavior, reflected in their support of and engagement with their children. Hence, H1 was largely confirmed.

Results also verified $\mathrm{H} 2$, indicating that the associations between the mothers' perceptions about the threat of COVID-19 and their lack of social and family support, and their children's externalizing and internalizing behaviors were mediated via the mothers' anxiety and hostile/coercive parenting behaviors, each on its own and consecutively.

The study's findings allude to the fact that the mothers' experience of the potential medical and financial threats of COVID-19 and the unavailability of social support following the social distancing regulations are meaningful for the mothers' emotional state and their parenting. In accordance with other studies conducted during COVID-19 (Malhotra et al., 2020; Romero et al., 2020), as well as during pre-COVID-19 disasters (Beaglehole et al., 2018), we found that the more the mother was threatened by COVID-19's short-term and long-term implications, the more anxious she was. Moreover, similar to previous studies (Chung et al., 2020; Romero et al., 2020), mothers' perceptions about the threat of COVID-19 were associated with negative maternal behaviors.

The lack of available social support, which resulted from the social distancing regulations, also emerged as a key factor in predicting the mothers' anxiety. These results accord with previous views of social support as vital to parents' wellbeing (Arikan et al., 2019) and specifically with recent findings during COVID-19 (Brown et al., 2020). Furthermore, the literature has consistently suggested the lack of social support as a risk factor for parenting behavior and practices (Rhoad-Drogalis et al., 2020). Our findings support this suggestion by showing the same pattern during COVID-19 in which lack of social support had a positive association with hostile/coercive parenting behaviors and a negative association with supportive/engaged maternal behaviors. The social distancing regulations and lockdowns that followed the spread of the pandemic substantially reduced the parents' and children's contact with their extended family, grandparents and friends. In so doing, the regulations limited actual and perceived support, which, as indicated above, are essential to parents in general, and in Israeli culture in particular.

In searching for the possible mechanisms that accounted for the links between the contextual features of COVID-19 and children's behavior problems, we found that the 
mothers' anxiety and their parenting behavior were mediators of these links, each on its own and sequentially. The mothers' anxiety emerged as a considerable risk factor, as it mediated the associations between COVID-19's contextual features and their children's behavior directly and indirectly, via hostile/coercive maternal behavior. This finding is consistent with the family stress model (Masrik \& Conger 2017) and in line with previous studies, suggesting that parents' anxiety can interfere with the family relationship and jeopardize the child's development (Pereira et al., 2014; MASKED, 2018). The worldwide evidence of the heightened anxiety associated with COVID-19 (Luo et al., 2020; Mazza et al., 2020) and the current findings linking the mothers' anxiety with COVID-19's contextual features suggest that the mothers' anxiety is central in understanding the pandemic's short and long-term consequences.

Interestingly, the mothers' anxiety mediated the association between their perceptions about the threat of COVID-19 and their children's internalizing behavior problems but not their externalizing behavior problems. It is possible that mothers who were more worried and concerned about the implications of COVID-19 were more focused on and attuned to their own fears (hence, the numerous reports of the mothers' anxiety), as well as their children's fears (hence, the numerous reports of internalizing behaviors) but less aware of their children's externalizing problems. In other words, anxious and worried mothers may project their own mental health difficulties on to the child, and/or may tend to view the child as more "fragile," thus overreporting their internalizing difficulties (Briggs-Gowan et al., 1996).

Moreover, the mothers' anxiety was linked to hostile/ coercive parenting behaviors. In addition, both factors mediated the association between the mothers' perceptions about the threat of COVID-19 and lack of social support and their children's externalizing and internalizing behavior. In other words, each of the COVID-19 contextual features was linked to more worries, fears and restlessness among the mothers. Their anxiety was linked with their hostile and coercive behavior towards their children, which, in turn, was associated with more maternal reports of children's externalizing and internalizing behaviors. Other studies from around the world during COVID-19 and the resulting quarantine documented a similar pattern (Romero et al., 2020; Spinelli et al., 2020; Whittle et al., 2020). This pattern is also consistent with previous disaster research, which showed that children's reactions to crises are at least partially determined by their families' response (Samuelson et al., 2017). The mediation pattern that emerged from our data can also be interpreted in light of the "spillover" hypothesis (Erel \& Burman, 1995), according to which negativity stemming from disruptions in one family subsystem may "spill over" into other subsystems. Thus, the mothers' anxiety may spill over into the parent-child interaction subsystem and "color" it with negativity, which is known to be a vital risk factor for children's socioemotional difficulties (Weymouth et al., 2016). This theoretical perspective, which serves as an explanation in routine times, is even more relevant in times of crisis, like the current, ongoing pandemic (Russell et al., 2020).

In our study, mothers' hostile/coercive behavior mediated the link between the lack of social support and the children's externalizing and internalizing behavior on its own. Previous studies have similarly noted the mediating role of hostile maternal behavior in various contexts (e.g., Coppola et al., 2020). It appears to be an important mediator, particularly when social support is lacking, which was the case during this pandemic (Clemens et al., 2020). The quarantine and social distancing regulations that followed the pandemic's outbreak forced mothers to cope with new, unusual challenges, without the support they were used to. Moreover, the lockdown of the school systems led mothers and children to spend a great deal of time together, confined to their homes, with working mothers simultaneously having to meet their own work demands, help their children with remote schooling tasks and provide them with indoor entertainment. Researchers have noted that these stressful and competing demands increase the burden on parents, and the risk for negative parenting behaviors and even child abuse (Ghosh et al., 2020). Thus, the multiple demands the mothers in our sample faced and the unavailability of support might have resulted in maternal impatience, fatigue and irritation. Under certain circumstances such as heightened anxiety, these factors might have increased the risk of negative parenting behavior toward the child, which, in turn, was associated with the children's behavioral problems.

Interestingly, the mediated associations between COVID-19's contextual features and the children's behavior problems via the mothers' positive, supportive, engaged parenting were only marginally significant. This finding differs from other studies conducted during the pandemic that did show associations between positive parenting and children's outcomes (Romero et al., 2020; Shen et al., 2020). It is possible that the inconsistency between the studies' findings is due to differences in their designs, the measures used and the reporters (e.g., children in Shen et al., 2020 vs. parents in our study). Moreover, previous research linked positive parenting with adaptive outcomes in children such as affect regulation capacities, empathy and prosocial responding (Davidov \& Grusec, 2006). In contrast, our study focused on children's maladaptation rather than positive adjustment, which may account for the lack of significant associations with positive parenting. In addition, the PBI was designed for use with preschoolers to school-aged children, which was the population of our study. We documented a negative association between supportive/engaged parenting behavior and the child's age. It is possible that using a narrower age range with younger 
children and larger sample would have resulted in additional significant findings regarding positive parenting. In sum, the potentially protective role of positive parenting in children's adjustment, both adaptive and maladaptive, during COVID-19 and in general, requires more exploration.

\section{Contributions and Limitations}

The current study joins and expands the emerging literature on COVID-19 in several ways. First, it proposes, tests and provides evidence for the specific parental mediating mechanisms that are associated with the worldwide reports of increased behavior problems in children in the context of COVID-19. Second, and relatedly, the study explores and highlights the importance of mothers' subjective experience of COVID-19 and how it relates to their mental health, parenting and children's behavior problems. Finally, the study is unique in its focus on relatively high SES families, demonstrating that even in this group, some mothers reported anxiety symptoms, hostile and coercive parenting behavior, and behavior problems in their children. Furthermore, the findings establish that these factors are interlinked. Thus, the study joins other emerging COVID-19 studies as well as the pre-COVID-19 literature that highlight the importance of the immediate context of caregivers as mediators and facilitators of children's socioemotional adaptation in general and during COVID-19 in particular.

Nevertheless, these contributions need to be viewed in light of some limitations. First, we had a relatively small final sample size, which might have limited our ability to detect additional effects. Moreover, our sample was relatively homogeneous in terms of the families' SES, the mothers' educational level and two-parent households. This homogeneity limits our ability to generalize the findings and draw conclusions about the implications of COVID-19 for other populations and family contexts. In addition, the children's age range in the study was relatively wide. Thus, it is possible that the mothers' parenting varied across different developmental stages, masking potential links with parenting. Further studies with specific reference to a narrower age range are needed to reveal information relevant to different stages of development. Furthermore, we collected our data concurrently, thus precluding causal, directional and reciprocal inferences. Whereas our underlying assumption was that maternal anxiety paves the way for negative parenting and behavior problems in children, it is equally possible that the children's difficult behavior leads to maternal anxiety and/or hostile parenting. Longitudinal designs with larger samples, focusing on both the children's strengths and weaknesses, are needed to test alternative models and further understand the family processes involved in this ongoing health crisis. In addition, we created the variables of COVID-19's contextual features ad hoc for the specific requirements of the current study. The reliabilities of these measurements were relatively low. Thus, additional studies with more extended and bettervalidated measures are needed. The fact that the study's measures were based on the mothers' reports also raises the risk of a shared method variance artifact. Finally, our study included mothers only. Additional studies that examine the role of fathers in children's socioemotional adaption in the context of COVID-19 are greatly needed. Notwithstanding these limitations, the study provides important insights into the mechanisms associated with the adjustment of mothers and children during the COVID-19 pandemic.

\section{Practical Implications}

COVID-19 is an ongoing, global crisis with extensive health, economic, social and psychological implications for children, parents and families that are only starting to be explored and understood. Our study's results underscore the importance of mothers' mental health and parenting practices for their children's socioemotional and behavioral adaption at this time. Thus, family practitioners and professionals working with children should think about ways to support children and parents during this prolonged, ongoing health crisis. Drawing on our findings, we can argue, albeit tentatively, that reducing the preoccupation with the COVID-19 threat, managing general anxiety and maintaining social connections may be helpful for the entire family. Hence, providing parents with information as to how to reduce COVID-19 related stress and anxiety and utilizing virtual, online and remote sources of social support can be beneficial for their own wellbeing and that of their children.

Acknowledgements We wish to thank Ravit Bachar and Inbar Ben Moshe for their help in recruitment and data collection. We also wish to thank the mothers who volunteered for the study for their time and effort.

\section{Compliance with Ethical Standards}

Conflict of Interest The authors declare no competing interests.

Publisher's note Springer Nature remains neutral with regard to jurisdictional claims in published maps and institutional affiliations.

\section{References}

Achenbach, T. M., \& Rescorla, L. A. (2000). Manual for the ASEBA preschool forms and profiles (Vol. 30). Burlington, VT: University of Vermont, Research Center for Children, Youth, \& Families.

Achenbach, T. M., \& Rescorla, L. A. (2001). Manual for the Achenbach system of empirically based assessment school-age forms profiles. Burlington, VT: Aseba. 
Arbuckle, J. L. (2019). Amos (version 26.0) [computer program]. IBM SPSS, Chicago.

Arikan, G., Kumru, A., Korkut, B., \& Ilhan, A. O. (2019). Examining toddlers' problem behaviors: The role of SES, parenting stress, perceived support and negative intentionality. Journal of Child and Family Studies, 28(12), 3467-3478. https://doi.org/10.1007/ s10826-019-01529-y.

Beaglehole, B., Mulder, R. T., Frampton, C. M., Boden, J. M., Newton-Howes, G., \& Bell, C. J. (2018). Psychological distress and psychiatric disorder after natural disasters: systematic review and meta-analysis. The British Journal of Psychiatry, 213(6), 716-722. https://doi.org/10.1192/bjp.2018.210.

Behrendt, H. F., Wade, M., Bayet, L., Nelson, C. A., \& Enlow, M. B. (2020). Pathways to social-emotional functioning in the preschool period: The role of child temperament and maternal anxiety in boys and girls. Development and Psychopathology, 32(3), 961-974.

Briggs-Gowan, M. J., Carter, A. S., \& Schwab-Stone, M. (1996). Discrepancies among mother, child, and teacher reports: Examining the contributions of maternal depression and anxiety. Journal of Abnormal Child Psychology, 24(6), 749-765.

Brown, S. M., Doom, J. R., Lechuga-Peña, S., Watamura, S. E., \& Koppels, T. (2020). Stress and parenting during the global COVID-19 pandemic. Child Abuse \& Neglect, 110(2), 104699. https://doi.org/10.1016/j.chiabu.2020.104699.

Chung, G., Lanier, P., \& Wong, P. Y. J. (2020). Mediating effects of parental stress on harsh parenting and parent-child relationship during coronavirus (COVID-19) pandemic in Singapore. Journal of Family Violence, 1-12.

Clemens, V., Deschamps, P., Fegert, J. M., Anagnostopoulos, D., Bailey, S., Doyle, M., \& Jacobs, B. (2020). Potential effects of" social" distancing measures and school lockdown on child and adolescent mental health. European Child \& Adolescent Psychiatry, 29, 739-742. https://doi.org/10.1007/s00787-020-01549-w.

Cobham, V. E., McDermott, B., Haslam, D., \& Sanders, M. R. (2016). The role of parents, parenting and the family environment in children's post-disaster mental health. Current Psychiatry Reports, 18(6), 53.

Cohen, S., \& McKay, G. (1984). Social support, stress and the buffering hypothesis: An empirical review. Handbook of Psychology and Health, 4, 253-267.

Condon, E. M., Holland, M. L., Slade, A., Redeker, N. S., Mayes, L. C., \& Sadler, L. S. (2019). Associations between maternal caregiving and child indicators of toxic stress among multiethnic, urban families. Journal of Pediatric Health Care, 33(4), $425-436$

Conger, R. D., \& Conger, K. J. (2002). Resilience in Midwestern families: Selected findings from the first decade of a prospective, longitudinal study. Journal of Marriage and Family, 64, 361-373. https://doi.org/10.1111/j.1741-3737.2002.00361.x.

Conger, R. D., Conger, K. J., \& Martin, M. J. (2010). Socioeconomic status, family processes, and individual development. Journal of Marriage and Family, 72, 685-704. https://doi.org/10.1111/j. 1741-3737.2010.00725.x.

Connor, J. J., \& Rueter, M. A. (2006). Parent-child relationships as systems of support or risk for adolescent suicidality. Journal of Family Psychology, 20(1), 143-155. https://psycnet.apa.org/doi/ 10.1037/0893-3200.20.1.143

Coppola, G., Costantini, A., Goffredo, M., Piazzolla, D. V. A., Semeraro, C., Cassibba, R., \& Mancini, F. (2020). The link between mother and child's obsessive-compulsive symptoms: A test of simple and serial mediation models in a healthy community sample. Journal of Obsessive-Compulsive and Related Disorders, 25, 100510 https://doi.org/10.1016/j.jocrd.2020.100510.

Davidov, M., \& Grusec, J. E. (2006). Untangling the links of parental responsiveness to distress and warmth to child outcomes. Child Development, 77(1), 44-58.
Derogatis, L. R. (1993). BSI Brief Symptom Inventory: Administration, scoring, and procedure manual (4th ed.). Minneapolis, MN: National Computer Systems.

Derogatis, L. R., \& Melisaratos, N. (1983). The Brief Symptom Inventory: An introductory report. Psychological Medicine, 13, 595-605.

Epkins, C. C., \& Harper, S. L. (2016). Mothers' and fathers' parental warmth, hostility/rejection/neglect, and behavioral control: Specific and unique relations with parents' depression versus anxiety symptoms. Parenting, 16(2), 125-145.

Erel, O., \& Burman, B. (1995). Interrelatedness of marital relations and parent-child relations: a meta-analytic review. Psychological Bulletin, 118(1), 108-132.

Gewirtz, A., Forgatch, M., \& Wieling, E. (2008). Parenting practices as potential mechanisms for child adjustment following mass trauma. Journal of Marital and Family Therapy, 34(2), 177-192. https://doi.org/10.1111/j.1752-0606.2008.00063.x.

Ghosh, R., Dubey, M. J., Chatterjee, S., \& Dubey, S. (2020). Impact of COVID-19 on children: Special focus on psychosocial aspect. Education, 31, 34.

Gibler, R. C., Kalomiris, A. E., \& Kiel, E. J. (2018). Paternal anxiety in relation to toddler anxiety: The mediating role of maternal behavior. Child Psychiatry \& Human Development, 49(4), 512-522.

Gil-Rivas, V., \& Kilmer, R. P. (2013). Children's adjustment following Hurricane Katrina: The role of primary caregivers. American Journal of Orthopsychiatry, 83, 413-421.

Griffith, A. K. (2020). Parental burnout and child maltreatment during the COVID-19 pandemic. Journal of Family Violence, 1-7.

Günther-Bel, C., Vilaregut, A., Carratala, E., Torras-Garat, S., \& PérezTestor, C. (2020). A mixed-method study of individual, couple, and parental functioning during the state-regulated COVID-19 lockdown in Spain. Family Process, 59(3), 1060-1079.

Halevi, G., Djalovski, A., Kanat-Maymon, Y., Yirmiya, K., ZagoorySharon, O., Koren, L., \& Feldman, R. (2017). The social transmission of risk: Maternal stress physiology, synchronous parenting, and well-being mediate the effects of war exposure on child psychopathology. Journal of Abnormal Psychology, 126(8), 1087 https://doi.org/10.1037/abn0000307.

Hentges, R. F., Graham, S. A., Plamondon, A., Tough, S., \& Madigan, S. (2019). A developmental cascade from prenatal stress to child internalizing and externalizing problems. Journal of Pediatric Psychology, 44(9), 1057-1067. https://doi.org/10.1093/jpepsy/jsz044.

Hu, L. T., \& Bentler, P. M. (1999). Cutoff criteria for fit indexes in covariance structure analysis: Conventional criteria versus new alternatives. Structural Equation Modeling: A Multidisciplinary Journal, 6(1), 1-55.

Imran, N., Zeshan, M., \& Pervaiz, Z. (2020). Mental health considerations for children \& adolescents in COVID-19 Pandemic. Pakistan Journal of Medical Sciences, 36(COVID19-S4), S67.

Israel Central Bureau of Statistics. (2008). Retrieved May 10, 2021 from https://www.cbs.gov.il/he/publications/DocLib/2008/seker_ hevrati06/pdf/h_print.pdf.

Jackson, Y., \& Warren, J. S. (2000). Appraisal, social support, and life events: Predicting outcome behavior in school-age children. Child Development, 71(5), 1441-1457. https://doi.org/10.1111/ 1467-8624.00238.

Jiao, W. Y., Wang, L. N., Liu, J., Fang, S. F., Jiao, F. Y., \& PettoelloMantovani, M., et al. (2020). Behavioral and emotional disorders in children during the COVID-19 epidemic. Journal of Pediatrics, 221, 264-266.e1. https://doi.org/10.1016/j.jpeds.2020.03.013.

Juth, V., Silver, R. C., Seyle, D. C., Widyatmoko, C. S., \& Tan, E. T. (2015). Post-disaster mental health among parent-child dyads after a major earthquake in Indonesia. Journal of Abnormal Child Psychology, 43(7), 1309-1318. https://doi.org/10.1007/s10802015-0009-8.

Katz, R., Lowenstein, A., Halperin, D., \& Tur-Sinai, A. (2015). Generational solidarity in Europe and Israel. Canadian Journal of 
Aging/La Revue Canadienne du Vieillissement, 34(3), 342-355. https://doi.org/10.1017/S0714980815000197.

Kelley, M. L., Self-Brown, S., Le, B., Bosson, J. V., Hernandez, B. C., \& Gordon, A. T. (2010). Predicting posttraumatic stress symptoms in children following Hurricane Katrina: A prospective analysis of the effect of parental distress and parenting practices. Journal of Traumatic Stress, 23(5), 582-590. https://doi.org/10.1002/jts.20573.

Lovejoy, M. C., Weis, R., O'Hare, E., \& Rubin, E. C. (1999). Development and initial validation of the Parent Behavior Inventory. Psychological Assessment, 11(4), 534-545.

Luo, M., Guo, L., Yu, M., Jiang, W., \& Wang, H. (2020). The psychological and mental impact of coronavirus disease 2019 (COVID-19) on medical staff and general public-A systematic review and meta-analysis. Psychiatry Research, 291, 113190.

Malhotra, C., Chaudhry, I., Ozdemir, S., \& Teo, I., \& Finkelstein, E. A. (2020). Anxiety and perceived risk during COVID-19 outbreak. medRxiv, https://doi.org/10.1101/2020.07.24.20161315.

Masarik, A. S., \& Conger, R. D. (2017). Stress and child development: A review of the Family Stress Model. Current Opinion in Psychology, 13, 85-90.

Mazza, C., Ricci, E., Biondi, S., Colasanti, M., Ferracuti, S., Napoli, C., \& Roma, P. (2020). A nationwide survey of psychological distress among Italian people during the COVID-19 pandemic: Immediate psychological responses and associated factors. International Journal of Environmental Research and Public Health, 17(9), 3165 https://doi.org/10.3390/ ijerph17093165.

Neubauer, A. B., Schmidt, A., Kramer, A. C., \& Schmiedek, F. (2021). A Little Autonomy Support Goes a Long Way: Daily AutonomySupportive Parenting, Child Well-Being, Parental Need Fulfillment, and Change in Child, Family, and Parent Adjustment Across the Adaptation to the COVID-19 Pandemic. Child Development, 1-19.

Orgilés, M., Morales, A., Delvecchio, E., Mazzeschi, C., \& Espada, J. P. (2020). Immediate psychological effects of the COVID-19 quarantine in youth from Italy and Spain. Frontiers in Psychology, 11, 2986.

Parkes, A., \& Sweeting, H. (2018). Direct, indirect, and buffering effects of support for mothers on children's socioemotional adjustment. Journal of Family Psychology, 32(7), 894-903. https://doi.org/10.1037/fam0000438.

Parkes, A., Sweeting, H., \& Wight, D. (2015). Parenting stress and parent support among mothers with high and low education. Journal of Family Psychology, 29(6), 907- 918. https://doi.org/ 10.1037/fam0000129.

Pereira, A. I., Barros, L., Mendonça, D., \& Muris, P. (2014). The relationships among parental anxiety, parenting, and children's anxiety: The mediating effects of children's cognitive vulnerabilities. Journal of Child and Family Studies, 23(2), 399-409.

Rhoad-Drogalis, A., Dynia, J. M., Justice, L. M., Purtell, K. M., Logan, J. A., \& Salsberry, P. J. (2020). Neighborhood Influences on Perceived Social Support and Parenting Behaviors. Maternal and Child Health Journal, 24(2), 250-258. https://doi.org/10. 1007/s10995-019-02861-x.

Romero, E., López-Romero, L., Domínguez-Álvarez, B., Villar, P., \& Gómez-Fraguela, J. A. (2020). Testing the effects of COVID-19 confinement in Spanish children: The role of parents' distress, emotional problems and specific parenting. International Journal of Environmental Research and Public Health, 17(19), 6975. https://doi.org/10.3390/ijerph17196975.

Rothenberg, W. A., Lansford, J. E., Alampay, L. P., Al-Hassan, S. M., Bacchini, D., Bornstein, M. H., \& Malone, P. S. (2020). Examining effects of mother and father warmth and control on child externalizing and internalizing problems from age 8 to 13 in nine countries. Development and Psychopathology, 32(3), 1-25. https://doi.org/10.1017/S0954579419001214.

Russell, B. S., Hutchison, M., Tambling, R., Tomkunas, A. J., \& Horton, A. L. (2020). Initial challenges of caregiving during COVID-19: Caregiver burden, mental health, and the parent-child relationship. Child Psychiatry \& Human Development, 51(5), 671-682.

Saddik, B., Hussein, A., Sharif-Askari, F. S., Kheder, W., Temsah, M.H., Koutaich, R. A., ... Halwani, R. (2020b). Increased levels of anxiety among medical and non-medical university students during the COVID-19 pandemic in the United Arab Emirates. medRvix. https://doi.org/10.1101/2020.05.10.20096933.

Samuelson, K. W., Wilson, C. K., Padron, E., Lee, S., \& Gavron, L. (2017). Maternal PTSD and children's adjustment: parenting stress and emotional availability as proposed mediators. Journal of Clinical Psychology, 73(6), 693-706. https://doi.org/10.1002/ jclp.22369.

Schwartz, O. S., Byrne, M. L., Simmons, J. G., Whittle, S., Dudgeon, P., Yap, M. B. \& Allen, N. B. (2014). Parenting during early adolescence and adolescent-onset major depression: A 6-year prospective longitudinal study. Clinical Psychological Science, 2(3), 272-286. https://doi.org/10.1177/2167702613505531.

Shen, Y., Luo, Z., Fu, D., Qie, M., \& Wang, L. (2020). Parenting profiles, intolerance of uncertainty, and worry among Chinese primary school children. Journal of Child and Family Studies, 29(8), 2303-2313.

Silverman, W. K., \& La Greca, A. M. (2002). Children experiencing disasters: Definitions, reactions, and predictors of outcomes. In A. M. L. Greca, W. K. Silverman, E. M. Vernberg, \& M. C. Roberts (Eds.), Helping children cope with disasters and terrorism. Washington, DC, US, American Psychological Association (pp. 11-33).

Slovic, P. (1987). Perception of risk. Science, 236(4799), 280-285.

Song, H. (2018). The longitudinal influence of maternal depression and anxiety on noncompliance and externalizing behavior problems of preschool children: The mediation effect of harsh maternal parenting behavior. Korean Journal of Child Studies, 39(1), 119-128. https://doi.org/10.5723/kjcs.2018.39.1.119.

Spinelli, M., Lionetti, F., Pastore, M., \& Fasolo, M. (2020). Parents' stress and children's psychological problems in families facing the COVID-19 outbreak in Italy. Frontiers in Psychology, 11, 1713 https://doi.org/10.3389/fpsyg.2020.01713.

Weymouth, B. B., Buehler, C., Zhou, N., \& Henson, R. A. (2016). A meta-analysis of parent-adolescent conflict: Disagreement, hostility, and youth maladjustment. Journal of Family Theory \& Review, 8(1), 95-112. https://doi.org/10.1111/jftr.12126.

Whittle, S., Bray, K. O., Lin, S., \& Schwartz, O. (2020). Parenting and child and adolescent mental health during the COVID-19 pandemic. PsyArXiv, https://doi.org/10.31234/osf.io/ag2r7.

Yap, M. B. H., Pilkington, P. D., Ryan, S. M., \& Jorm, A. F. (2014). Parental factors associated with depression and anxiety in young people: A systematic review and meta-analysis. Journal of Affective Disorders, 156, 8-23. https://doi.org/10.1016/j.jad. 2013.11.007.

Zhou, S. J., Zhang, L. G., Wang, L. L., Guo, Z. C., Wang, J. Q., Chen, J. C., \& Chen, J. X. (2020). Prevalence and socio-demographic correlates of psychological health problems in Chinese adolescents during the outbreak of COVID-19. European Child \& Adolescent Psychiatry, 29(6), 749-758.

Zilber, N., Auerbach, J., \& Lerner, Y. (1994). Israeli norms for the Achenbach Child Behavior Checklist: Comparison of clinically referred and non-referred children. Israel Journal of Psychiatry and Related Sciences, 31(1), 5-12. 